\title{
Reduced cognitive deficits after FLASH irradiation of whole mouse brain are associated with less hippocampal dendritic spine loss and neuroinflammation
}

Danielle A. Simmons ${ }^{\mathrm{a}}$; Frederick M. Lartey ${ }^{\mathrm{b}, \mathrm{c}}$; Emil Schüler ${ }^{\mathrm{b}}$; Marjan Rafat ${ }^{\mathrm{b}, \mathrm{d}}$; Gregory

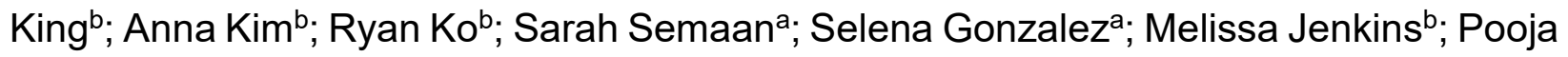
Pradhan'; Zion Shih ${ }^{\mathrm{b}}$; Jinghui Wang ${ }^{\mathrm{b}}$; Rievon Eyben ${ }^{\mathrm{b}}$; Edward E. Graves ${ }^{\mathrm{b}, \mathrm{e}}$; Peter G.

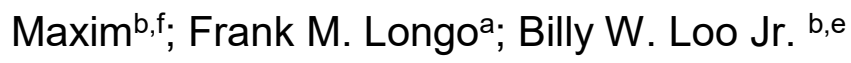

a. Department of Neurology and Neurological Sciences, , Stanford University School of Medicine, United States

b. Department of Radiation Oncology, Stanford University School of Medicine, United States

c. The Jackson Laboratory, Sacramento, United States

d. Department of Chemical and Biomolecular Engineering, Vanderbilt University, Nashville, United States

e. Stanford Cancer Institute, Stanford University School of Medicine, United States

f. Department of Radiation Oncology, Indiana University School of Medicine, Indianapolis, United States

This is the author's manuscript of the article published in final edited form as:

Simmons, D. A., Lartey, F. M., Schüler, E., Rafat, M., King, G., Kim, A., Ko, R., Semaan, S., Gonzalez, S., Jenkins, M., Pradhan, P., Shih, Z., Wang, J., von Eyben, R., Graves, E. E., Maxim, P. G., Longo, F. M., \& Loo, B. W. (2019). Reduced cognitive deficits after FLASH irradiation of whole mouse brain are associated with less hippocampal dendritic spine loss and neuroinflammation. Radiotherapy and Oncology, 139, 4-10. https://doi.org/10.1016/j.radonc.2019.06.006 


\title{
HIGHLIGHTS
}

- Brain radiation impairs cognition, associated with neuronal degeneration and neuroinflammation.

- Ultra-rapid FLASH produced reduced cognitive deficits vs. conventional delivery time.

- Loss of hippocampal dendritic spines and neuroinflammation were less evident after FLASH.

- These factors may mediate the improved therapeutic index of FLASH brain irradiation.

\section{ABSTRACT}

\begin{abstract}
AIM
To evaluate the impact of ultra-rapid FLASH mouse whole brain irradiation on hippocampal dendritic spines and neuroinflammation, factors associated with cognitive impairment after brain irradiation.

\section{Methods}

We administered $30 \mathrm{~Gy}$ whole brain irradiation to C57BL6/J mice in sub-second (FLASH) vs. $240 \mathrm{~s}$ conventional delivery time keeping all other parameters constant, using a custom configured clinical linac. Ten weeks post-irradiation, we evaluated spatial and non-spatial object recognition using novel object location and object recognition testing. We measured dendritic spine density by tracing Golgi-stained hippocampal neurons and evaluated neuroinflammation by CD68 immunostaining, a marker of activated microglia, and expression of 10 pro-inflammatory cytokines using a multiplex immunoassay.
\end{abstract}

\section{RESULTS}

At ten weeks post-irradiation, compared to unirradiated controls, conventional delivery time irradiation significantly impaired novel object location and recognition tasks whereas the same dose given in FLASH delivery did not. Conventional delivery time, but not FLASH, was associated with significant loss of dendritic spine density in hippocampal 
apical dendrites, with a similar non-significant trend in basal dendrites. Conventional delivery time was associated with significantly increased CD68-positive microglia compared to controls whereas FLASH was not. Conventional delivery time was associated with significant increases in 5 of 10 pro-inflammatory cytokines in the hippocampus (and non-significant increases in another 3), whereas FLASH was associated with smaller increases in only 3.

\section{CONCLUSION}

Reduced cognitive impairment and associated neurodegeneration were observed with FLASH compared to conventional delivery time irradiation, potentially through decreased induction of neuroinflammation, suggesting a promising approach to increasing therapeutic index in radiation therapy of brain tumors.

Keywords: Whole brain irradiation; Radiation-induced cognitive impairment; FLASH; Ultra-rapid radiation

Radiation therapy is an important treatment modality for a broad range of brain tumors. However, the ability to cure tumors through dose intensity is limited by normal tissue injury, particularly when a large volume of the brain (up to the whole brain) must be 
included in the treatment volume as is the case for many clinical indications. In the prospective randomized trial RTOG $0614,64.9 \%$ of patients developed a significant decrement in cognitive function by 24 weeks after whole brain radiation therapy [1]. In this study, the addition of memantine as a neuroprotective agent reduced the incidence of cognitive failure to $53.8 \%$. That hippocampal radiation injury is implicated in the observed cognitive impairment is supported by the prospective randomized trial NRG Oncology CC001 in which whole brain irradiation with conformal dose reduction to the hippocampus combined with memantine reduced cognitive failure at 6 months compared to standard whole brain radiation and memantine, from $68.2 \%$ to $59.5 \%$ in this study [8]. Despite both neuroprotection and hippocampal dose reduction, the incidence of significant radiationinduced cognitive decline remains over $50 \%$.

Preclinical models replicate the observations in humans in that whole brain irradiation in rodents produces measurable chronic cognitive deficits, and these deficits are associated with loss of hippocampal neurogenesis and loss of dendritic spine density in hippocampal neurons [12], [2], [18], [4]]. These effects appear to be mediated by neuroinflammation, particularly microglial activation, which demonstrates a strong radiation dose-response and can be at least partially mitigated by anti-inflammatory interventions [13]. Activated microglia mediate the secretion of pro-inflammatory cytokines, including interleukin (IL)$1 \beta$, IL-6, and tumor necrosis factor alpha (TNF $\alpha$ ) in the hippocampus and other brain areas following cranial irradiation [7], [10], [23], [6].

Recently, preclinical studies of ultra-rapid "FLASH" radiation delivery have demonstrated unexpected sparing of normal organ injury compared to conventional dose rate delivery of the same doses, while maintaining equal tumoricidal effects [5], [22]. In the brain, FLASH irradiation has been shown to spare hippocampal neurogenesis and non-spatial memory in mice [14], [15]. FLASH therefore represents a promising new approach to increasing substantially the therapeutic index of radiation therapy solely through modifying radiation delivery, but the fundamental mechanisms underlying this effect remain poorly understood.

Using a customized configuration of a clinical high-energy electron linear accelerator to enable FLASH irradiation in small animals with favorable dosimetric characteristics [19], 
we sought to evaluate whether sparing of cognition by FLASH whole brain irradiation is associated with corresponding sparing of the integrity of hippocampal dendritic spines and decreased neuroinflammation. We isolated total delivery time as the dosimetric variable between FLASH and conventional delivery time irradiation, keeping other parameters including dose per pulse, number of pulses, irradiation geometry, and beam type and energy the same.

\section{MATERIALS AND METHODS}

\section{STUDY DESIGN}

We assessed whether ultra-rapid (FLASH) whole brain irradiation could mitigate the neurodegeneration, neuroinflammation, and associated cognitive deficits observed with conventional delivery time irradiation. Mice from a single breeding source ( 3 months of age) were randomly assigned to one of three experimental groups $(n=21-26$ mice per group): 1. control (no irradiation); 2. conventional delivery time irradiation; and 3. FLASH irradiation. Approximately 10 weeks after irradiation, each cohort underwent object location and novel object recognition testing to assess the effects on learning and memory. Twenty-four hours after behavioral testing, mice were euthanized and their brains harvested for morphological and biochemical analyses. We used immunohistochemistry to assess the development of radiation-induced microglial activation, Golgi staining to determine dendritic spine density, and a multiplex immunoassay to determine cytokine levels.

\section{ANIMALS}

Three-month-old male C57BL6/J mice (Jackson Laboratory, Sacramento, CA) were housed at the Stanford Research Animal Facility on a 12/12 light-dark cycle with food and water provided ad libitum. Animal care was conducted in accordance with the NIH Guide for the Care and Use of Laboratory Animals and this study was approved by the Institutional Animal Care and Use Committee of Stanford University.

\section{BRAIN IRRADIATION}


Whole brain irradiation was performed using a custom configuration of a clinical linear accelerator (Varian Clinac 21EX, Varian Medical Systems, Palo Alto, CA) using an electron beam [19]. Fig. 1 depicts the irradiation geometry. Briefly, C57BL6/J mice were anesthetized by intraperitoneal injection of ketamine and immobilized in a custom acrylic stereotactic positioning frame, constructed for reproducible alignment of the mouse skull relative to a lead collimator to shape the beam to cover the whole brain and block the eyes. A lateral beam was used to irradiate the whole brain while sparing the pharyngeal axis. Gafchromic EBT2 films (Ashland Inc, Covington, KY) were placed in the stereotactic frame to record the entrance dose for every mouse. The electron beam energies used were 16 and $20 \mathrm{MeV}$. The relatively uniform depth dose across the width of the mouse brain (Fig. 1) of beams with these energies in this irradiation geometry was previously characterized [19]. The nominal radiation dose was taken to be the mean entrance dose over the irradiation field recorded on film for each mouse. We targeted delivery of $30 \mathrm{~Gy}$ radiation dose to each mouse brain in a single fraction in delivery times of either $0.1-$ $0.16 \mathrm{~s}$ (FLASH, with average dose rate 200 or $300 \mathrm{~Gy} / \mathrm{s}$ for 20 or $16 \mathrm{MeV}$ beams, respectively) or $240 \mathrm{~s}$ (conventional delivery time, with average dose rate $0.13 \mathrm{~Gy} / \mathrm{s}$ for both 16 and $20 \mathrm{MeV}$ beams). In both FLASH and conventional delivery time, the dose was delivered in a train of pulses (18 pulses on average) of $2 \mu \mathrm{s}$ duration, each with an average dose per pulse of $1.75 \mathrm{~Gy}$ and an average intra-pulse dose rate of $8.75 \times 10^{5} \mathrm{~Gy} / \mathrm{s}$. The delivery time was varied by controlling the pulse rate: 108 (for $20 \mathrm{MeV}$ beam) or 180 (for $16 \mathrm{MeV}$ beam) pulses per second for FLASH; and 5 trains of 2-3 pulses each (delivered at a rate of 18 pulses per second for both 16 and $20 \mathrm{MeV}$ beams) separated by $60 \mathrm{~s}$ for a total delivery time of $240 \mathrm{~s}$ for conventional delivery time. 


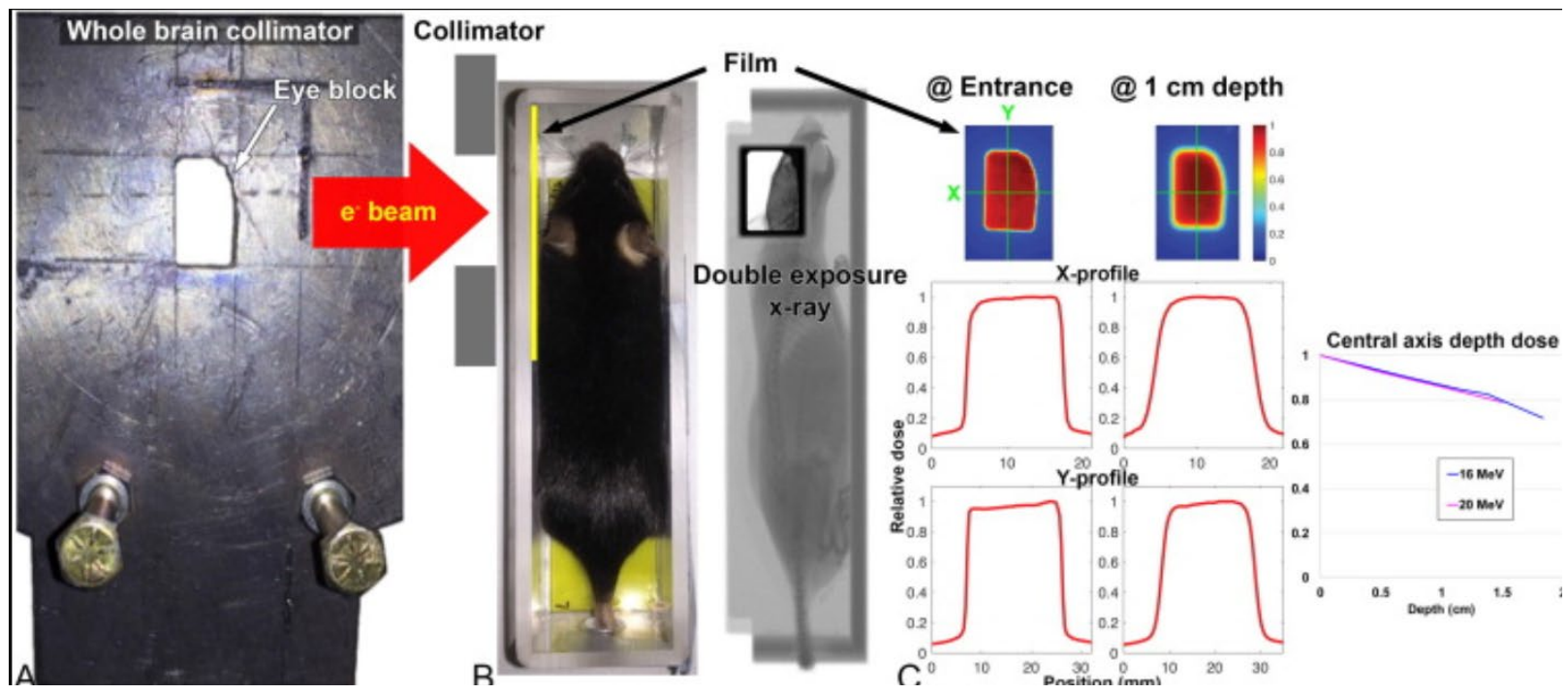

Fig. 1. Irradiation geometry. (A) A lead collimator shapes the electron radiation field to cover the whole brain while blocking the eyes and pharyngeal axis. (B) The mouse is placed in a stereotactic positioning frame and the whole brain is irradiated via a lateral field (left), with film measurement of entrance dose for every irradiation. A double exposure lateral radiograph (right) demonstrates alignment of the collimated beam relative to the mouse. $30 \mathrm{~Gy}$ whole brain irradiation was administered in either $0.1-0.16 \mathrm{~s}$ (FLASH) or $240 \mathrm{~s}$ (conventional delivery time) by varying the pulse rate, keeping all other parameters constant. (C) Films at the beam entrance and at $1 \mathrm{~cm}$ depth in a solid water phantom exposed at $20 \mathrm{MeV}$ electron energy, with corresponding transverse dose profiles, demonstrate dose homogeneity throughout the field (left). Central axis depth dose measured in a solid water phantom is relatively constant over the depth of the brain at both 16 and $20 \mathrm{MeV}$ electron energy (right).

\section{OBJECT LOCATION AND NOVEL OBJECT RECOGNITION TESTING}

On the habituation day and the following 2 test days, mice were acclimated to the dimly lit testing room for $30 \mathrm{~min}$ in their home cages. They were also habituated to the empty $45 \times 38 \times 28 \mathrm{~cm}$ testing arena covered by a white cloth for $10 \mathrm{~min}$ the day before testing. Object location: the ability of the mice to detect the new location of a familiar object was assessed on Testing Day 1 (Fig. 2A). Mice were placed in the arena with 2 unfamiliar objects positioned $5 \mathrm{~cm}$ away from the walls and were allowed to explore the arena and the objects for $10 \mathrm{~min}$ (Training). One hour after training, one of the familiar objects was 
moved to a new location and mice were again allowed to explore for $10 \mathrm{~min}$. Novel object recognition: twenty-four hours later (Testing Day 2), the familiar object that remained in the same location on Testing Day 1 was replaced with a novel object. Mice were allowed to explore the arena and the objects for $10 \mathrm{~min}$ [11]. The arena and objects were cleaned with $70 \%$ ethanol between each mouse exploration session and each session was recorded using a video recorder attached to the side of the arena. The duration of time spent exploring each object, defined as sniffing with the head within $1 \mathrm{~cm}$ of the object, was recorded by an experimenter blind to the treatment conditions. Mice were excluded if they failed to explore the objects for more than the a priori threshold of one minute during the training session. A discrimination index was calculated using the following equation: discrimination index $=[$ (novel object or location exploration time - familiar object or location exploration time)/total time spent exploring objects] ${ }^{\star 100}$. Statistical significance of difference between treatment groups was determined using a one-way ANOVA with Fisher's LSD post-hoc test.

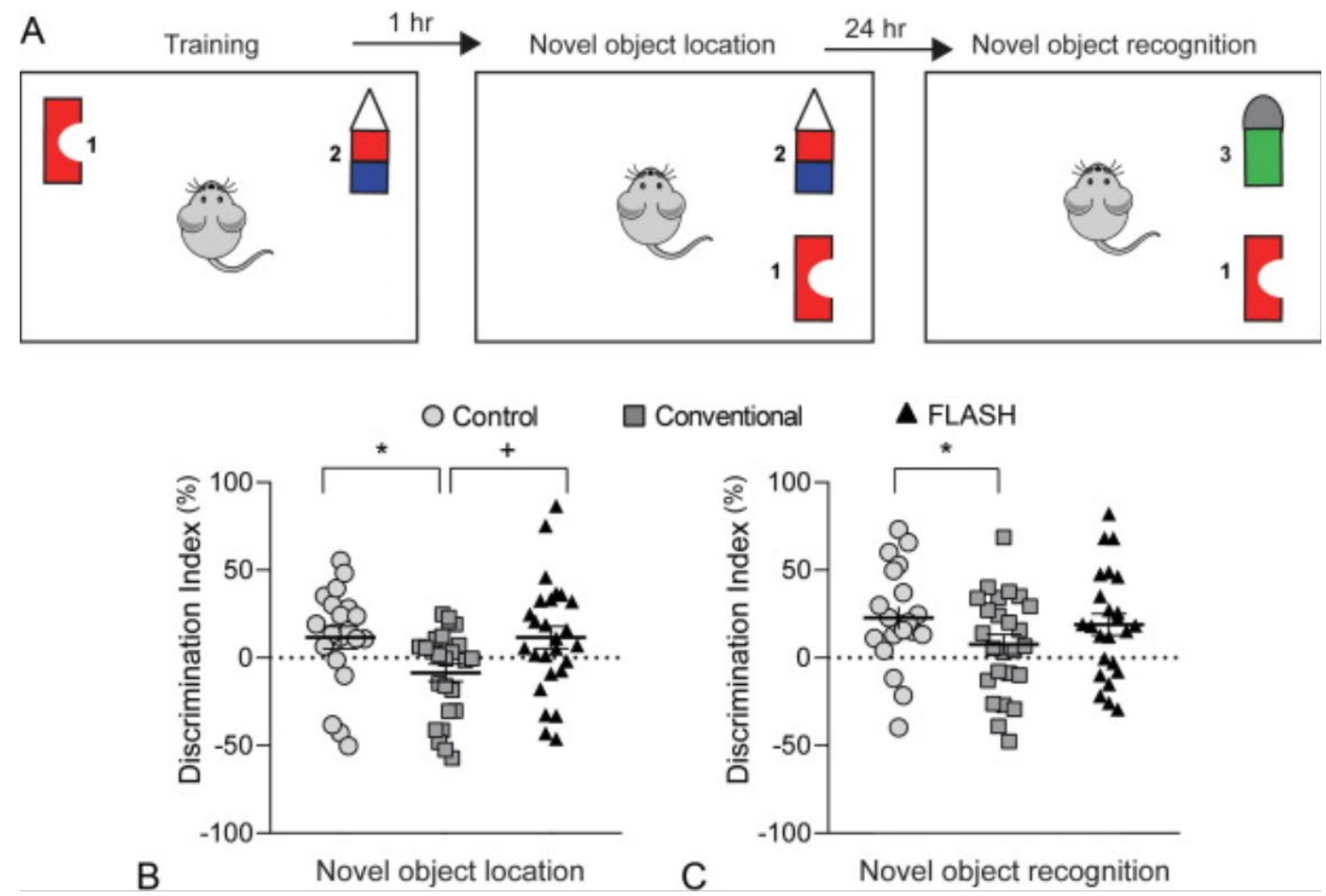

Fig. 2. FLASH irradiation is associated with reduced deficits in spatial and non-spatial object recognition 10 weeks post-irradiation. (A) Schema of novel object location and novel object recognition assays. (B) Novel object location: mice given conventional 
delivery time irradiation $(n=25)$ spent significantly less time with a familiar object in a novel location than control mice $\left(n=20 ;{ }^{*} p=0.017\right)$. Mice given FLASH irradiation $(n=26)$ performed similarly to controls and explored the novel location significantly more than conventional delivery time irradiated mice $\left({ }^{+} p=0.049\right)$, indicating sparing of hippocampus-dependent spatial learning and memory. (C) Novel object recognition: mice given conventional delivery time irradiation explored a novel object significantly less than controls $\left({ }^{*} p=0.04\right)$ while those given FLASH irradiation performed similarly to controls $(p=0.67)$; novel object recognition was non-significantly decreased after conventional delivery time irradiation compared to FLASH $(p=0.17)$. Discrimination index $=[($ novel object or location exploration time - familiar object or location exploration time)/total time spent exploring objects] ${ }^{*} 100 \%$. Statistical significance of differences between treatment groups was determined using a one-way ANOVA with Fisher's LSD post-hoc test. Results are plotted as mean \pm SEM.

\section{MODIFIED GOLGI STAINING AND QUANTITATION OF DENDRITIC SPINE DENSITY}

Modified Golgi staining was performed as described previously [21], [20] on one brain hemisphere from each mouse of one cohort of mice. Briefly, brains were immersed in modified Golgi-Cox staining solution (purchased from Drs. Deqiang Jing and Francis Lee at Cornell University) for 9 days. They were transferred to $30 \%$ sucrose in $\mathrm{dH}_{2} \mathrm{O}$ at $4{ }^{\circ} \mathrm{C}$ for $72 \mathrm{~h}$. Brains were then cut into $150 \mu \mathrm{m}$ sections with a vibratome while immersed in $30 \%$ sucrose at room temperature. Sections were mounted onto slides coated with $0.3 \%$ gelatin, dipped in $40 \%$ sucrose 3 times, and allowed to air dry for $72 \mathrm{~h}$. Sections were washed with $\mathrm{dH}_{2} \mathrm{O}$, stained with developing solution, dehydrated with graded ethanols, immersed in histoclear, and then coverslipped using DPX mounting medium.

Dendritic spine density was determined by manually tracing all branches on all dendritic trees of Golgi-stained CA1 pyramidal cells in the dorsal hippocampus while viewing and adjusting focus at 100x using Neurolucida neuron tracing software (MBF Bioscience, Williston, VT). Three neurons per mouse that minimally overlapped adjacent neurons and did not appear to be truncated prematurely due to sectioning were selected for tracing. Neurolucida Explorer software (MBF Bioscience) was used to determine the density of spines on dendritic branches of third order and higher as first and second order dendrites 
have very few spines. Statistical significance of differences between treatment groups was determined using a one-way ANOVA with Fisher's LSD post-hoc test.

\section{HISTOLOGY AND IMMUNOHISTOCHEMISTRY FOR MICROGLIAL ACTIVATION}

Immunohistochemistry (IHC) for CD68 (a specific marker for activated microglia) was performed as follows: mice were euthanized with carbon dioxide and perfused with phosphate-buffered saline (PBS). The brains were then harvested, embedded and frozen in OCT (Sakura Fintek, Torrance, CA, USA) using dry ice and stored at $-80^{\circ} \mathrm{C}$ until $8 \mu \mathrm{m}$ sections were cut with a cryostat onto slides. The primary antibody used was rat monoclonal anti-mouse CD68 (Abcam, Cambridge, MA, USA), diluted at 1:1000 in the blocking solution. The secondary antibody used was goat anti-rat IgG Alexa Fluor 488 (Invitrogen, Carlsbad, CA, USA). The staining procedure was based on standard IHC techniques for frozen sections. Briefly, the sections were placed in paraformaldehyde (4\%) for $15 \mathrm{~min}$ and then in PBS containing $\mathrm{NH}_{4} \mathrm{Cl}(50 \mathrm{mM})$ for $5 \mathrm{~min}$. They were subsequently transferred into chilled methanol-acetone $\left(1: 1\right.$ at $\left.-20^{\circ} \mathrm{C}\right)$ for $5 \mathrm{~min}$, followed immediately by Triton-X 100 (0.1\% in PBS) for 5 min. Blocking was performed with $5 \%$ goat serum in PBS containing $0.5 \%$ Tween 20 for 30 min and immediately incubated with the primary antibody at room temperature for $1 \mathrm{~h}$ followed by incubation with the fluorescent dye-labeled secondary antibody, also for $1 \mathrm{~h}$. The sections were washed extensively with PBS after every step. They were then mounted in DAPI-containing medium and imaged with an epifluorescence microscope (Carl Zeiss Inc., Oberkochen, Germany). For each of 6 mice per treatment group (unirradiated control, conventional delivery time, and FLASH), two hemi-brain coronal sections through the hippocampus were imaged by automated acquisition of intermediate-power fields (IPFs, 20x) that were stitched together to form the whole image of each section. Images were analyzed with ImageJ software (https://imagej-nih-gov.proxy.ulib.uits.iu.edu/ij/index.html). CD68positive cells were counted in every 20x IPF covering the hippocampus on each section, approximately 36-40 IPFs per section, by an observer blinded to the treatment group. The total number of CD68-positive cells $( \pm S E M)$ was scored for each mouse in each treatment group. Statistical significance of differences between treatment groups was determined using a one-way ANOVA with Fisher's LSD post-hoc test. 


\section{CYTOKINE ASSAY}

After perfusion with saline and brain extraction, the hippocampus was dissected from one brain hemisphere per mouse in the analysis cohorts and flash frozen at $-80^{\circ} \mathrm{C}$ until use for cytokine measurement. Flash frozen hippocampi were sonicated in RIPA lysis buffer containing protease and phosphatase inhibitors. Levels of 10 cytokines were measured in hippocampal lysates from each treatment group run in duplicate using a V-PLEX Proinflammatory Panel 1 Mouse Kit (Meso Scale Diagnostics, Rockville, MD), which is a multiplex sandwich immunoassay with electrochemiluminescence (ECL), according to the kit instructions. Discovery Workbench 4.0 software (Meso Scale Diagnostics) was used to generate a calibration curve to which ECL signals were fit to determine analyte concentrations.

\section{RESULTS}

The measured entrance dose based on film dosimetry was $30.8 \pm 0.09$ Gy (mean \pm SEM) for conventional delivery time and $30.4 \pm 0.37 \mathrm{~Gy}$ for FLASH irradiation.

Regarding the impact of FLASH and conventional delivery time irradiation on cognitive function, ten weeks post-irradiation the performance of mice on spatial and non-spatial variants of the object recognition task was assessed. This unsupervised learning task exploits the animal's innate proclivity to explore novel environmental stimuli. The spatial variant is hippocampus-dependent and involves discerning a familiar object in a novel location. One mouse was removed from the control group and one mouse was removed from the conventional delivery time irradiation group since they failed to explore the objects for more than the a priori threshold of one minute during the training session.

Mice irradiated with conventional delivery time spent significantly less time exploring the familiar object in the novel location compared to non-irradiated control mice (Fig. 2B). This memory impairment was reduced in mice exposed to FLASH irradiation as their exploration of the object in the novel location was significantly greater than mice with conventional delivery time irradiation and did not differ significantly from controls (Fig. 2B). The non-spatial variant of the task is standard novel object recognition and the brain areas involved in mediating this task include the perirhinal and prefrontal cortices. 
Conventional delivery time irradiation significantly decreased the ability of the mice to distinguish the novel object from the familiar one (Fig. 2C). The discrimination index after FLASH irradiation was not significantly different from controls, though the increase over conventional delivery time also did not reach statistical significance. All mice explored the objects for similar amounts of time during training, indicating that mobility or bias/preference were not likely contributors to the differential exploration of object/location during testing. The times spent exploring objects during training were (mean $\pm S E M$ ): unirradiated controls, $89.1 \pm 9.7 \mathrm{~s}$; conventional delivery time, $77.1 \pm 5.6 \mathrm{~s}$; FLASH, $78.7 \pm 9.3 \mathrm{~s}$. Thus, FLASH irradiation may be particularly effective in sparing hippocampus-dependent spatial learning and memory.

Dendritic spines are highly plastic and critical for memory formation. Conventional delivery time radiation has been shown to decrease dendritic spines in the dentate gyrus of the rat hippocampus [2], [18], [4]. Given that FLASH irradiation had hippocampusdependent memory sparing effects, we investigated its effects on spine density of apical and basal dendrites of CA1 pyramidal neurons, as these are the major output cells of the hippocampus (Fig. 3A). CA1 apical dendrites integrate spatial and contextual information for transmission to other brain regions. At ten weeks post-irradiation, compared to unirradiated controls, spine density of apical dendrites was significantly reduced by $18 \pm 4.9 \%$ with conventional delivery time but not FLASH irradiation (Fig. $3 \mathrm{~B}$ and $\mathrm{C}$ ), though the increase in apical dendritic spine density with FLASH over conventional delivery time did not reach statistical significance. The spine density of basal dendrites after FLASH was intermediate between those of controls and conventional delivery time, but these differences did not reach statistical significance (Fig. $3 \mathrm{C}$ ). 


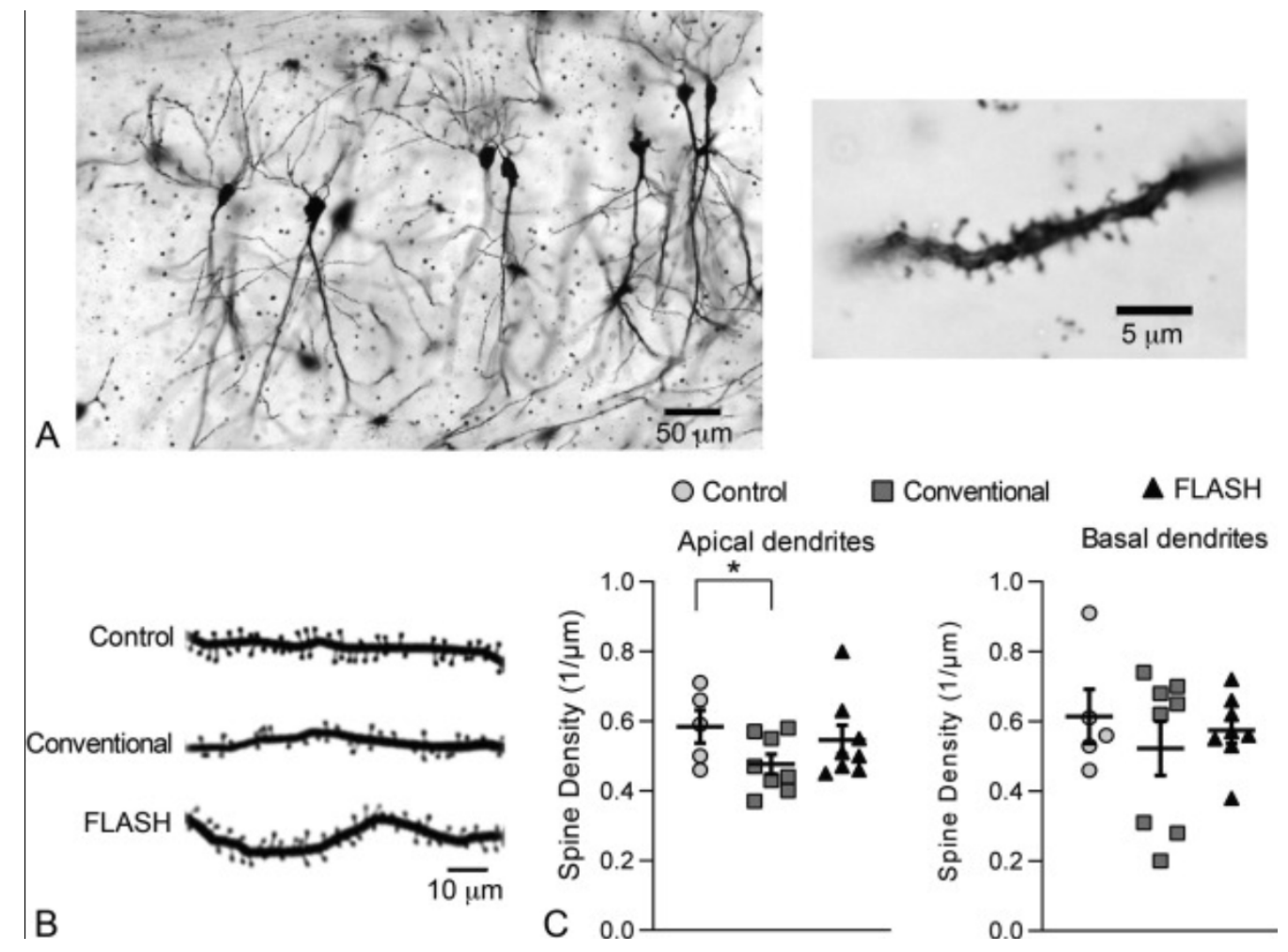

Fig. 3. FLASH irradiation is associated with reduced dendritic spine loss in the hippocampus 10 weeks post-irradiation. (A) Left: representative low power (10x) image of Golgi staining of pyramidal neurons in the CA1 hippocampal region of a mouse that received FLASH irradiation. Scale bar $=50 \mu \mathrm{m}$. Right: representative high power $(100 \times)$ image of Golgi-stained spines of an apical dendrite of a CA1 pyramidal neuron in the hippocampus of a mouse that received FLASH irradiation. Scale bar $=5 \mu \mathrm{m}$. (B) Representative tracings of hippocampal neurons demonstrate loss of dendritic spine density after conventional delivery time irradiation but relative sparing after FLASH irradiation. (C) Compared to unirradiated controls ( $n=5$ mice), conventional delivery time irradiation ( $n=8$ mice) significantly decreased the spine density on apical (center) but not basal (right) dendrites of CA1 pyramidal neurons of the hippocampus $\left({ }^{*} p=0.03\right)$. FLASH irradiation ( $n=8$ mice) did not significantly decrease spine density compared to controls and apical dendritic spine density was higher with FLASH compared to conventional delivery time irradiation, but this difference was not statistically significant $(p=0.08)$. The density of spines was determined for dendritic branches of third order and higher. Statistical significance of differences between treatment groups was determined using a one-way ANOVA with Fisher's LSD post-hoc test. Results are plotted as mean \pm SEM. 
Microglial activation is an index of neuroinflammation. At ten weeks post-irradiation, the microglial protein CD68, a biomarker of microglial activation, was expressed at significantly higher levels in conventional delivery time irradiation vs. unirradiated control mouse brains (Fig. 4A), whereas in FLASH it was intermediate between control and conventional delivery time. Mean counts of CD68-positive cells per mouse were 132.2 \pm 58.4 (mean \pm SEM) for unirradiated controls, significantly increased to $379 \pm 45.5$ for conventional delivery time $(p=0.0036)$, and nonsignificantly increased to $255.4 \pm 47.9$ for FLASH ( $p=0.12$ vs. control; $p=0.12$ vs. conventional delivery time, Fig. 4B).

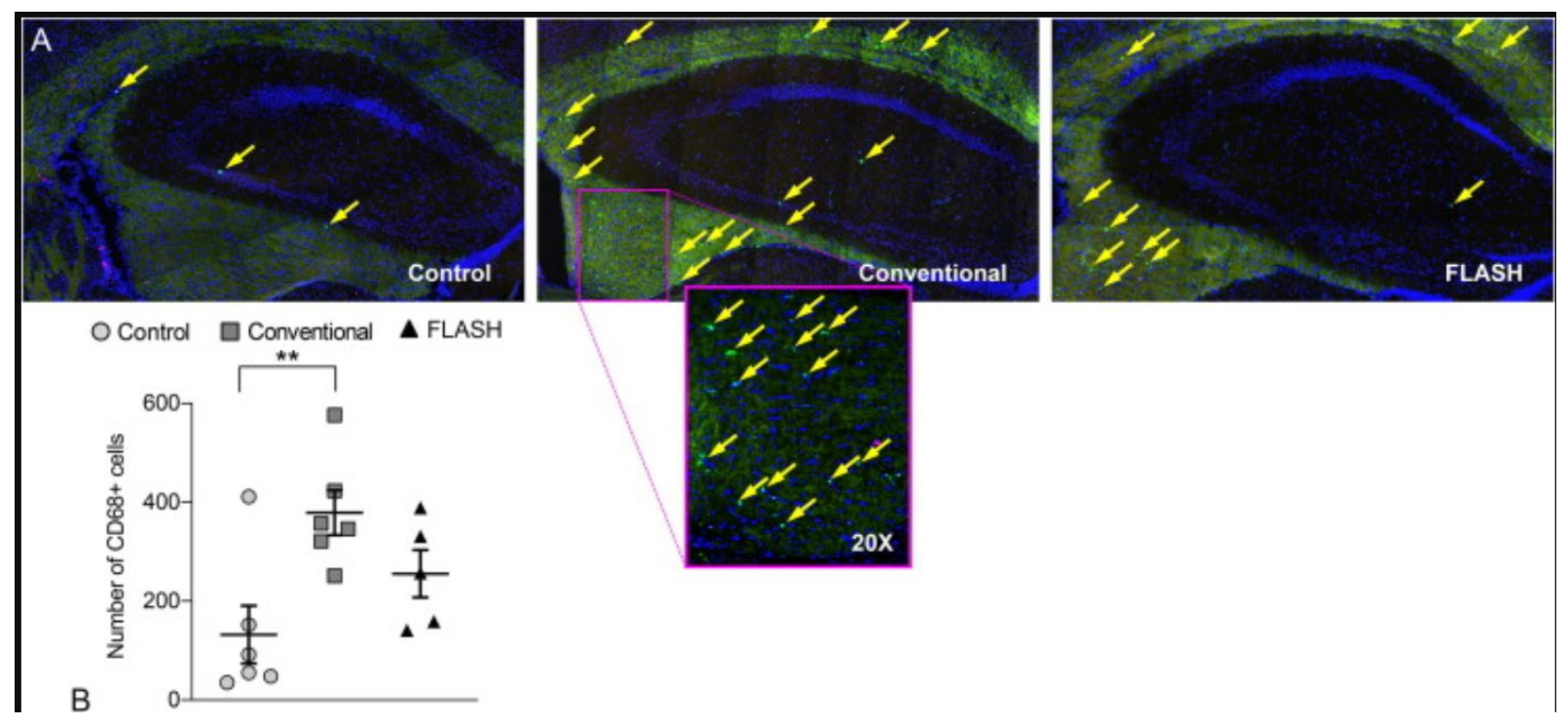

Fig. 4. FLASH irradiation induces less microglial activation 10 weeks post-irradiation. (A) Representative sections through the hippocampus with nuclei stained by DAPI (blue) and CD68-positive activated microglia appearing as green foci (arrows). Compared to the unirradiated control group, conventional delivery time irradiation significantly increased microglial activation, whereas FLASH was intermediate between control and conventional delivery time. (B) Quantification of CD68-positive cells per hippocampal section ( $n=6$ mice each for control and conventional delivery time; $n=5$ for FLASH) demonstrating significantly increased neuroinflammation after conventional delivery time irradiation compared to control $\left({ }^{* *} p=0.0036\right)$, but non-significantly increased after FLASH ( $p=0.12$ vs. control; $p=0.12$ vs. conventional delivery time). Statistical significance of differences between treatment groups was determined using a one-way ANOVA with Fisher's LSD post-hoc test. Results are plotted as mean \pm SEM. The neuroinflammation induced in human brain tissue by conventional delivery time radiotherapy involves 
increased microglial expression of cytokines including IL-6 and TNFa [7], [10], [23], [6]. Initially such cytokines may be released to protect neurons but when up-regulated chronically they can contribute to delayed radiation neurodegeneration including dendritic spine loss and cognitive dysfunction. We measured a panel of ten pro-inflammatory cytokine concentrations in the hippocampus. Compared to unirradiated controls, conventional delivery time irradiation was associated with statistically significant increases in levels of 5 of the 10 cytokines (IL-6, IL-1 $\beta$, TNFa, KC/GRO, and IL-4), nonsignificant increases in another 3 (IL-2, IL-5, and IL-12), and no change in IFN- $\gamma$ and IL10 (Fig. 5). In contrast, FLASH irradiation was associated with significant increases in only 3 of the 10 cytokines (IL-1 $\beta, T N F \alpha, K C / G R O$ ) over controls, and to a lesser degree than conventional delivery time. IL-6 and IL-1 $\beta$ levels were significantly lower and $\mathrm{KC} / \mathrm{GRO}$ levels trended lower $(p=0.07)$ with FLASH than with conventional delivery time. Another 5 cytokines were non-significantly lower with FLASH than with conventional delivery time (TNFa, IL-4, IL-2, IL-5, and IL-12). Overall, FLASH was associated with decreased neuroinflammation compared to conventional delivery time as demonstrated by reductions in microglial activation (CD68) and levels of certain key pro-inflammatory cytokines.

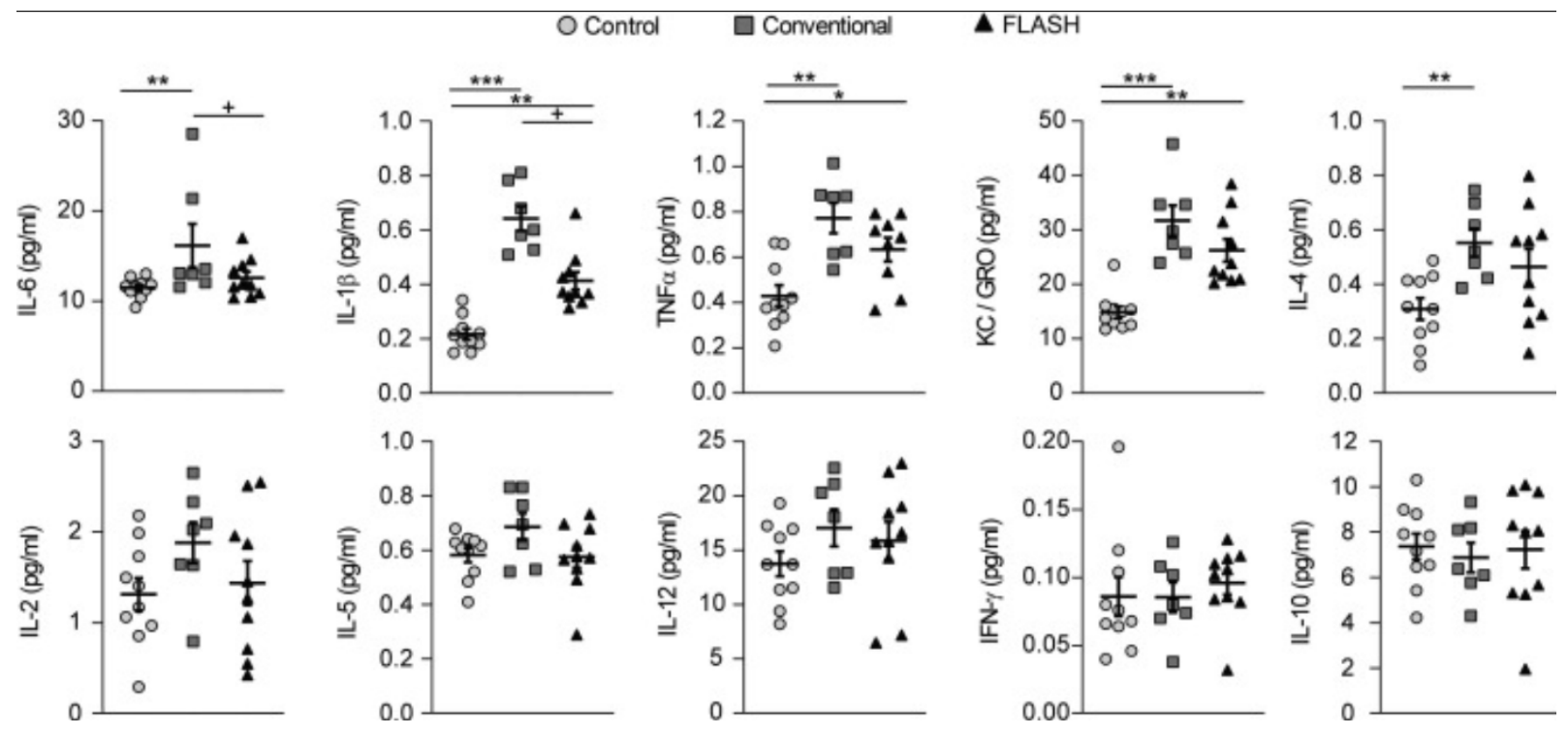

Fig. 5. FLASH irradiation induces lower pro-inflammatory cytokine levels in the hippocampus 10 weeks post-irradiation. Compared to unirradiated controls ( $n=10$ mice), conventional delivery time irradiation ( $n=7$ mice) was associated with statistically 
significant increases in levels of 5 of the 10 cytokines tested (IL-6, IL-1 $\beta, T N F \alpha, K C / G R O$, and IL-4) and non-significant increases in another 3 (IL-2, IL-5, and IL-12). In contrast, FLASH irradiation ( $n=10$ mice) was associated with significant increases in only 3 of the 10 cytokines (IL-1 $\beta, \mathrm{TNF} \alpha, \mathrm{KC} / \mathrm{GRO}$ ) over controls, and to a lesser degree than conventional delivery time. IL-6 and IL-1 $\beta$ levels were significantly lower and KC/GRO levels trended lower $(p=0.07)$ with FLASH than with conventional delivery time. Another 5 cytokines were non-significantly lower with FLASH than with conventional delivery time (TNFa, IL-4, IL-2, IL-5, and IL-12). ${ }^{*} p \leq 0.05,{ }^{* *} p \leq 0.01$, and ${ }^{* * *} p \leq 0.001$ compared to controls. ${ }^{+} p<0.05$ compared to conventional delivery time irradiation. Statistical significance of differences between treatment groups was determined using a one-way ANOVA with Fisher's LSD post-hoc test. Results are plotted as mean \pm SEM. Discussion We demonstrated in this study that, consistent with prior studies [14], [15], [16], FLASH is associated with reduced cognitive deficits compared to conventional delivery time irradiation. Concomitant with this functional finding was the observation that the loss of hippocampal dendritic spines was similarly reduced with FLASH. Our findings that FLASH appeared to be associated with less activation of microglial inflammation and decreased hippocampal expression of multiple cytokine mediators of inflammation suggest that the differential effects on normal tissues of FLASH compared to conventional delivery time irradiation occur at the level of induction of inflammatory responses, leading to sparing of the common downstream processes impacting cognitive function.

Distinguishing technical aspects of our study include the use of a high-energy $16-20 \mathrm{MeV}$ electron beam [19], which while similar in nature overall to prior platforms used for FLASH studies [9], has an advantage of more uniform depth dose characteristics because of the higher beam energy. For experimental tractability, we chose to focus on a limited set of parameters to study. In particular, it is unclear which aspect of rapid delivery is the most important for producing the FLASH biological phenomenon, as it can be a combination of factors including average dose rate, total delivery time, dose per pulse, pulse rate, intrapulse dose rate, etc. We chose to isolate delivery time, keeping the beam type and energy, irradiation geometry, dose per pulse, and intra-pulse dose rate constant, 
changing only the pulse rate (and concomitantly overall delivery time and average dose rate) between FLASH and conventional delivery time.

Similarly, we chose to evaluate only one high single fraction dose (30 Gy) where we anticipated robust inflammatory, neuroanatomical, and cognitive responses with conventional delivery time, and evaluated all endpoints at one time point (10 weeks postirradiation). We also focused only on normal tissue rather than tumor effects. Of note, while this is a very high single fraction whole brain radiation dose compared to what is clinically tolerable in humans, it has been demonstrated in previous studies to be tolerable in mice with minimal side effects (provided the pharyngeal axis is excluded from the high dose radiation field) at least within the first 2-3 months, exceeding the 10-week observation period of our experiments [3], [17].

An additional limitation of our study is small sample sizes that constrained the statistical power to detect differences in a number of the endpoints. Nevertheless, the insights gained from this study will inform future investigations into the phenomenological and underlying mechanistic aspects of FLASH irradiation.

In conclusion, our study demonstrated that compared to conventional delivery time

irradiation, FLASH irradiation was associated with reduced cognitive deficits, potentially through ameliorating neuroinflammation and consequently less loss of hippocampal dendritic spine density. Further elucidation of the mechanisms underlying these effects will facilitate ultimate clinical translation of a new strategy for improving the therapeutic index of radiation therapy for cancer.

\section{DECLARATION OF COMPETING INTEREST}

Drs. Loo, Maxim, and Graves have received research support from Varian Medical Systems. Dr. Maxim is a co-founder and Dr. Loo is a co-founder and board member of TibaRay.

\section{ACKNOWLEDGEMENTS}


This work was supported by the Stanford University Department of Radiation Oncology, the Weston Havens Foundation, the Stanford University Department of Neurology and Neurological Sciences, the Stanford University School of Medicine, the Stanford University Office of the Provost, the SLAC National Accelerator Laboratory, the Wallace H. Coulter Foundation, the Jean Perkins Foundation, the Cancer League, the Swedish Childhood Cancer Foundation, the Foundation BLANCEFLOR Boncompagni Ludovisi n'ee Bildt, and the American Association for Cancer Research.

The authors would like to thank Miguel Jimenez, Daniel Pawlak, James Clayton, and Flavio Poehlmann from Varian Medical Systems for their technical assistance on the FLASH irradiation system. 


\section{REFERENCES}

1. Brown, P. D., Pugh, S., Laack, N. N., Wefel, J. S., Khuntia, D., Meyers, C., Choucair, A., Fox, S., Suh, J. H., Roberge, D., Kavadi, V., Bentzen, S. M., Mehta, M. P., \& WatkinsBruner, D. (2013). Memantine for the prevention of cognitive dysfunction in patients receiving whole-brain radiotherapy: A randomized, double-blind, placebo-controlled trial. Neuro-Oncology, 15(10), 1429-1437. https://doi.org/10.1093/neuonc/not114

2. Chakraborti, A., Allen, A., Allen, B., Rosi, S., \& Fike, J. R. (2012). Cranial Irradiation Alters Dendritic Spine Density and Morphology in the Hippocampus. PLOS ONE, 7(7), e40844. https://doi.org/10.1371/journal.pone.0040844

3. Chiang, C.-S., McBride, W. H., \& Rodney Withers, H. (1993). Myelin-associated changes in mouse brain following irradiation. Radiotherapy and Oncology, 27(3), 229-236. https://doi.org/10.1016/0167-8140(93)90079-N

4. Ding, X., Wu, H.-H., Ji, S.-J., Cai, S., Dai, P.-W., Xu, M.-L., Zhang, J.-J., Zhang, Q.-X., Tian, Y., \& Ma, Q.-H. (2017). The p75 neurotrophin receptor regulates cranial irradiationinduced hippocampus-dependent cognitive dysfunction. Oncotarget, 8(25), 4054440557. https://doi.org/10.18632/oncotarget. 16492

5. Favaudon, V., Caplier, L., Monceau, V., Pouzoulet, F., Sayarath, M., Fouillade, C., Poupon, M.-F., Brito, I., Hupé, P., Bourhis, J., Hall, J., Fontaine, J.-J., \& Vozenin, M.-C. (2014). Ultrahigh dose-rate FLASH irradiation increases the differential response between normal and tumor tissue in mice. Science Translational Medicine, 6(245), 245ra93-245ra93. https://doi.org/10.1126/scitranslmed.3008973

6. Furuse, M., Nonoguchi, N., Kawabata, S., Miyatake, S.-I., \& Kuroiwa, T. (2015). Delayed brain radiation necrosis: Pathological review and new molecular targets for treatment. Medical Molecular Morphology, 48(4), 183-190. https://doi.org/10.1007/s00795-015$\underline{0123-2}$

7. Gaber, M. W., Sabek, O. M., Fukatsu, K., Wilcox, H. G., Kiani, M. F., \& Merchant, T. E. (2003). Differences in ICAM-1 and TNF- $\alpha$ expression between large single fraction and fractionated irradiation in mouse brain. International Journal of Radiation Biology, 79(5), 359-366. https://doi.org/10.1080/0955300031000114738 
8. V. Gondi, S. Deshmukh, P.D. Brown, et al.Preservation of neurocognitive function (NCF) with conformal avoidance of the hippocampus during whole-brain radiotherapy (HAWBRT) for brain metastases: preliminary results of phase III trial NRG Oncology CC001

9. Int J Radiat Oncol Biol Phys, 102 (2018), p. 1607

10. Jaccard, M., Durán, M. T., Petersson, K., Germond, J.-F., Liger, P., Vozenin, M.-C., Bourhis, J., Bochud, F., \& Bailat, C. (2018). High dose-per-pulse electron beam dosimetry: Commissioning of the Oriatron eRT6 prototype linear accelerator for preclinical use. Medical Physics, 45(2), 863-874. https://doi.org/10.1002/mp.12713

11. Lee, W. H., Sonntag, W. E., Mitschelen, M., Yan, H., \& Lee, Y. W. (2010). Irradiation induces regionally specific alterations in pro-inflammatory environments in rat brain. International Journal of Radiation Biology, 86(2), 132-144. https://doi.org/10.3109/09553000903419346

12. Miedel, C. J., Patton, J. M., Miedel, A. N., Miedel, E. S., \& Levenson, J. M. (2017). Assessment of Spontaneous Alternation, Novel Object Recognition and Limb Clasping in Transgenic Mouse Models of Amyloid- $\beta$ and Tau Neuropathology. JoVE (Journal of Visualized Experiments), 123, e55523. https://doi.org/10.3791/55523

13. Mizumatsu, S., Monje, M. L., Morhardt, D. R., Rola, R., Palmer, T. D., \& Fike, J. R. (2003). Extreme Sensitivity of Adult Neurogenesis to Low Doses of X-Irradiation. Cancer Research, 63(14), 4021-4027.

14. Monje, M. L., Toda, H., \& Palmer, T. D. (2003). Inflammatory Blockade Restores Adult Hippocampal Neurogenesis. Science, 302(5651), 1760-1765. https://doi.org/10.1126/science.1088417

15. Montay-Gruel, P., Petersson, K., Jaccard, M., Boivin, G., Germond, J.-F., Petit, B., Doenlen, R., Favaudon, V., Bochud, F., Bailat, C., Bourhis, J., \& Vozenin, M.-C. (2017). Irradiation in a flash: Unique sparing of memory in mice after whole brain irradiation with dose rates above 100Gy/s. Radiotherapy and Oncology, 124(3), 365-369. https://doi.org/10.1016/j.radonc.2017.05.003

16. Montay-Gruel, P., Bouchet, A., Jaccard, M., Patin, D., Serduc, R., Aim, W., Petersson, K., Petit, B., Bailat, C., Bourhis, J., Bräuer-Krisch, E., \& Vozenin, M.-C. (2018). X-rays can trigger the FLASH effect: Ultra-high dose-rate synchrotron light source prevents 
normal brain injury after whole brain irradiation in mice. Radiotherapy and Oncology, 129(3), 582-588. https://doi.org/10.1016/j.radonc.2018.08.016

17. Montay-Gruel, P., Acharya, M. M., Petersson, K., Alikhani, L., Yakkala, C., Allen, B. D., Ollivier, J., Petit, B., Jorge, P. G., Syage, A. R., Nguyen, T. A., Baddour, A. A. D., Lu, C., Singh, P., Moeckli, R., Bochud, F., Germond, J.-F., Froidevaux, P., Bailat, C., ... Limoli, C. L. (2019). Long-term neurocognitive benefits of FLASH radiotherapy driven by reduced reactive oxygen species. Proceedings of the National Academy of Sciences, 116(22), 10943-10951. https://doi.org/10.1073/pnas.1901777116

18. Moravan, M. J., Olschowka, J. A., Williams, J. P., \& O’Banion, M. K. (2011). Cranial Irradiation Leads to Acute and Persistent Neuroinflammation with Delayed Increases in T-Cell Infiltration and CD11c Expression in C57BL/6 Mouse Brain. Radiation Research, 176(4), 459-473. https://doi.org/10.1667/RR2587.1

19.Parihar, V. K., \& Limoli, C. L. (2013). Cranial irradiation compromises neuronal architecture in the hippocampus. Proceedings of the National Academy of Sciences, 110(31), 12822-12827. https://doi.org/10.1073/pnas.1307301110

20. Schüler, E., Trovati, S., King, G., Lartey, F., Rafat, M., Villegas, M., Praxel, A. J., Loo, B. W., \& Maxim, P. G. (2017). Experimental Platform for Ultra-high Dose Rate FLASH Irradiation of Small Animals Using a Clinical Linear Accelerator. International Journal of Radiation Oncology ${ }^{*}$ Biology ${ }^{*}$ Physics, $\quad 97(1), \quad$ 195-203. https://doi.org/10.1016/j.ijrobp.2016.09.018

21. Simmons, D. A., Belichenko, N. P., Ford, E. C., Semaan, S., Monbureau, M., Aiyaswamy, S., Holman, C. M., Condon, C., Shamloo, M., Massa, S. M., \& Longo, F. M. (2016). A small molecule p75NTR ligand normalizes signalling and reduces Huntington's disease phenotypes in R6/2 and BACHD mice. Human Molecular Genetics, 25(22), 4920-4938. https://doi.org/10.1093/hmg/ddw316

22. Simmons, D. A., Belichenko, N. P., Yang, T., Condon, C., Monbureau, M., Shamloo, M., Jing, D., Massa, S. M., \& Longo, F. M. (2013). A Small Molecule TrkB Ligand Reduces Motor Impairment and Neuropathology in R6/2 and BACHD Mouse Models of Huntington's Disease. Journal of Neuroscience, 33(48), 18712-18727. https://doi.org/10.1523/JNEUROSCI.1310-13.2013 
23. Vozenin, M.-C., Fornel, P. D., Petersson, K., Favaudon, V., Jaccard, M., Germond, J.-F., Petit, B., Burki, M., Ferrand, G., Patin, D., Bouchaab, H., Ozsahin, M., Bochud, F., Bailat, C., Devauchelle, P., \& Bourhis, J. (2019). The Advantage of FLASH Radiotherapy Confirmed in Mini-pig and Cat-cancer Patients. Clinical Cancer Research, 25(1), 35-42. https://doi.org/10.1158/1078-0432.CCR-17-3375

24. Yoritsune, E., Furuse, M., Kuwabara, H., Miyata, T., Nonoguchi, N., Kawabata, S., Hayasaki, H., Kuroiwa, T., Ono, K., Shibayama, Y., \& Miyatake, S.-I. (2014). Inflammation as well as angiogenesis may participate in the pathophysiology of brain radiation necrosis. Journal of Radiation Research, 55(4), 803-811. https://doi.org/10.1093/jrr/rru017 